$63^{\text {ème }}$ Congrès de la SFCO, 01001 (2015)

DOI:10.1051/sfco/20156301001

(C) Owned by the authors, published by EDP Sciences, 2015

\title{
CONFÉRENCE
}

\section{Les cellules souches mésenchymateuses de la moelle osseuse et des tissus adipeux : de la physiologie à la médecine régénératrice}

Casteilla L

STROMALab, UMR5273 UPS/CNRS, EFS Inserm U1031, BP. 84 225, 31432 Toulouse, France

louis.casteilla@inserm.fr

Les cellules souches mésenchymateuses (CSM) de la moelle osseuse ont été identifiées pour la première fois par Alexandre Friedenstein dans les années 1960.

Ces cellules furent alors décrites comme des précurseurs mésenchymateux capables de se différencier non seulement en ostéoblastes, mais également en chondrocytes et en adipocytes. Rapidement, il est aussi apparu qu'elles pouvaient participer à différents microenvironnements et soutenir ainsi le maintien de population de cellules souches, leur prolifération et agir sur leur devenir et différenciation. Bien que qualifiées de cellules souches, ce qui impliquerait que les CSM soient capables d'autorenouvellement, cette dernière propriété n’a pas été vraiment démontrée ce qui laisse une ambiguïté sur le terme à utiliser. Il a aussi été montré que les CSM avaient une très forte activité paracrine dans laquelle on peut inclure la sécrétion de multiples molécules capable de moduler l'inflammation et l'immunité.

Des cellules similaires ont été depuis décrites dans la majorité des tissus et particulièrement dans les tissus adipeux, elles sont alors appelées ASC (pour « adipose derived stroma/stem cells »).

Etant donné leur multipotence ainsi que leur activité paracrine, les différents types de CSM ont été testés dans un très grand nombre de modèles murins de dégénération tissulaire. Cependant, comme la fréquence de ces cellules dans les tissus adipeux est très élevée et ce tissu facile à prélever, de plus en plus d'études utilisent cette source tissulaire.

En fait, bien que très similaires, les CSM de la moelle osseuse et les ASC présentent des différences d'efficacité en fonction des domaines. Ainsi si les CSM de moelle se différencient mieux en ostéoblastes et chondrocytes pour un protocole donné, les ASC présentent des activités adipogéniques, angiogéniques et immunomodulatrices plus efficaces.

In vivo, deux problèmes importants restent à résoudre pour expliquer leur fonctionnement et efficacité après leur transplantation. Premièrement, il apparaît de plus en plus que les effets thérapeutiques sont faiblement corrélés au nombre de cellules différenciées issues des CSM transplantées. Deuxièmement, il est extrêmement difficile de démontrer à long terme la présence des CSM transplantées alors que les effets persistent.

Au niveau clinique, les CSMs furent utilisées pour la première fois pour traiter avec succès une pathologie de l'os : l'ostéogenèse imparfaite. Une recherche bibliographique sur le site clinicaltrial.gov montre clairement que le nombre d'essais cliniques ne cesse depuis d'augmenter. Bien que pour certaines applications, ces essais soient en phase 4, l'efficacité avérée chez l'homme est le plus souvent en cours d'évaluation. Leurs résultats dans les années à venir décideront de l'importance et de l'étendue que l'on pourra donner à ce nouveau champ thérapeutique. Quoiqu'il en soit, il apparaît d’ores et déjà :

1) que ces cellules jouent un rôle fondamental dans l'homéostasie tissulaire qui reste à mieux comprendre pour bien le maitriser,

2) qu’il existe déjà des applications où leur usage apporte un véritable bénéfice thérapeutique.

This is an Open Access article distributed under the terms of the Creative Commons Attribution License 4.0, which permits unrestricted use, distribution, and reproduction in any medium, provided the original work is properly cited. 